\section{Commentary: Through the looking glass: Deciding who we want to be}

Uma M. Sachdeva, MD, PhD, and

Yolonda L. Colson, MD, PhD

Despite our rich history of scientific advancements made by cardiothoracic surgeons, ranging from hyperkalemic cardiac arrest and cardiopulmonary bypass to heparin prophylaxis for deep vein thrombosis, the number of cardiothoracic surgeons with independent academic research funding remains markedly low. The reasons for this are multifactorial, reflecting clinical demands on time, limited funding to support research at both the institutional and national levels, and lack of formal pathways and infrastructure to support cardiothoracic surgeons pursuing investigative research early in their careers. ${ }^{1}$ In this issue of the Journal, Fudulu and Angelini ${ }^{2}$ describe structural advancements in policy, education, and attitudes in the United Kingdom that support the training and academic career trajectory of cardiac surgeons to foster involvement in research from medical school to professorship. Through National Health Service-affiliated programs, a centralized national training model has been created that provides protected research time at all stages of training and academic career development, at the same time integrating these research periods into the clinical training model, which Fudulu and Angelini ${ }^{2}$ describe as a "product pipeline" to create academic surgeons. The establishment of clear steps that lead to a path in academic surgery indicates a national commitment to creating, fostering, and maintaining surgeon-investigators. Moreover, they describe a national funding initiative that supports research by reimbursing hospitals for participation in research that is aligned with national health

From the Division of Thoracic Surgery, Massachusetts General Hospital and Harvard Medical School, Boston, Mass.

Disclosures: The authors reported no conflicts of interest.

The Journal policy requires editors and reviewers to disclose conflicts of interest and to decline handling or reviewing manuscripts for which they may have a conflict of interest. The editors and reviewers of this article have no conflicts of interest.

Received for publication July 27, 2020; revisions received July 27, 2020; accepted for publication July 27, 2020; available ahead of print Aug 3, 2020.

Address for reprints: Yolonda L. Colson, MD, PhD, Division of Thoracic Surgery, Massachusetts General Hospital, 55 Fruit St, Founders 7, Boston, MA 02114 (E-mail: ycolson@mgh.harvard.edu).

J Thorac Cardiovasc Surg 2021;162:1596-7

0022-5223/ $\$ 36.00$

Copyright $₫ 2020$ Published by Elsevier Inc. on behalf of The American Association for Thoracic Surgery

https://doi.org/10.1016/j.jtcvs.2020.07.080

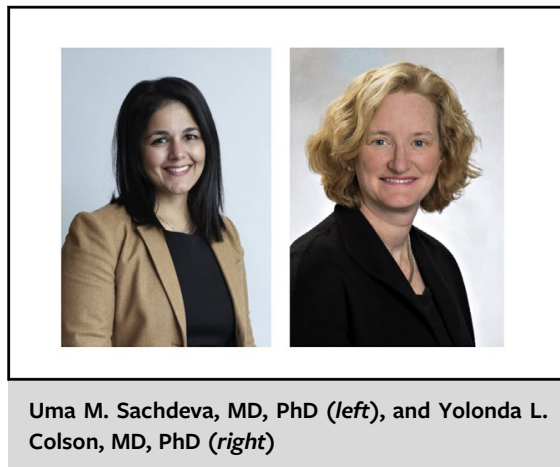

CENTRAL MESSAGE

The United Kingdom has estab-

lished infrastructural support at

the national level in a concerted

effort to address challenges and

develop cardiac surgeon-

investigators.

care goals and priorities, thereby establishing a hospital culture in which research and the surgeons who perform research have not only intellectual but also financial value. Despite these major advances, continued efforts are needed to safeguard continued academic progression, as Fudulu and Angelini ${ }^{2}$ report only 2 cardiac surgeons who have achieved the rank of associate professor in the past 5 years.

Despite the prominence of academic centers in the United States, demands for clinical productivity have endangered the surgeon-investigator. If we are to encourage young surgeons to pursue such careers and make the necessary commitments of time and effort when the pressures of clinical productivity and family responsibilities are greatest, it is critical to demonstrate that being a surgeonscientist is not just theoretically possible but realistically attainable and personally rewarding. ${ }^{3}$ The appointment of surgeon-investigators to leadership positions, both within the institution and in professional organizations, is crucial to recruit and maintain surgeons in this track. Without appropriate role models, robust institutional support, and assistance in navigating the challenges of competitive national funding and ultimate progression to full professorship and positions of national leadership, it is difficult for young surgeons to feel encouraged to pursue this career path, which is so fundamental to the future of our specialty. The United Kingdom has taken the initiative to support and develop both the clinical and research talents of cardiac 
surgeons to create a more secure and sustainable future for the specialty. This article of Fudulu and Angelini ${ }^{2}$ provides an opportunity for all of us to take a few minutes from our administrative and clinical duties to ask whether we are building the future we want for our specialty and are truly equipping our young surgeons with the necessary skills and opportunities to get us there.

\section{References}

1. Ikonomidis JS, Menasché P, Kreisel D, Sellke FW, Woo YJ, Colson YL. Attrition of the cardiothoracic surgeon-scientist: definition of the problem and remedial strategies. J Thorac Cardiovasc Surg. 2019;158:504-8.

2. Fudulu DP, Angelini GD. Flourish or perish: the UK academic surgeon model. $J$ Thorac Cardiovasc Surg. 2021;162:1590-4.

3. Goldstein AM, Blair AB, Keswani SG, Gosain A, Morowitz M, Kuo JS, et al. A roadmap for aspiring surgeon-scientists in today's healthcare environment. Ann Surg. 2019;269:66-72 